\title{
Estimação de parâmetros genéticos para características de crescimento de ovinos Santa Inês utilizando modelos uni e multicaracterísticas
}

[Estimation of genetic parameters for growth traits of Santa Inês sheep using single and multiple-trait models]

\author{
J.L.R. Sarmento ${ }^{1,4}$, R.A. Torres ${ }^{2,4}$, W.H. Sousa ${ }^{3}$, C.S. Pereira ${ }^{5}$, P.S. Lopes $^{2,4}$, F.C. Breda ${ }^{1}$ \\ ${ }^{1}$ Aluno de pós-graduação - UFV \\ ${ }^{2}$ Departamento de Zootecnia - UFV \\ Av. P.H. Rolfs, s/n \\ 36570-000 - Viçosa, MG \\ ${ }^{3} \mathrm{EMEPA}-\mathrm{PB}$ \\ ${ }^{4}$ Bolsista do CNPq \\ ${ }^{5}$ Bolsista da FAPEMIG
}

\begin{abstract}
RESUMO
Utilizaram-se registros de pesos do nascimento aos 196 dias de idade de 927 cordeiros, filhos de 45 reprodutores e 323 matrizes de ovinos da raça Santa Inês, controlados de 1983 a 2000, com o objetivo de avaliar três modelos que consideraram ou não o efeito genético materno e a (co)variância entre os efeitos genéticos direto e materno, para estimar componentes de variância e parâmetros genéticos por meio de modelos uni e bicaracterísticas. Os componentes de (co)variâncias e os parâmetros genéticos direto e materno para os pesos foram estimados pelo método da máxima verossimilhança restrita, sob modelo animal. De acordo com o teste de razão de verossimilhança, o modelo que incluiu o efeito aditivo direto mais o efeito materno foi o indicado para todas as características estudadas. A não-inclusão do efeito materno no modelo de análise superestimou as variâncias e as herdabilidades para o efeito direto $(0,56 \mathrm{a}$ 0,23). A importância do efeito materno diminuiu ao longo da trajetória de crescimento, à medida que a idade dos cordeiros aumentava. As variâncias e as herdabilidades estimadas por meio dos modelos bicaracterísticas para os efeitos genéticos diretos foram superiores às obtidas pelos modelos unicaracterísticas. As correlações genéticas entre as características foram altas e positivas. O efeito materno foi importante para todas as características estudadas, devendo, portanto, ser considerado nos estudos de crescimento. Os modelos bicaracterísticas possibilitaram resgatar parte da variância aditiva direta, levando a estimativas maiores de herdabilidade.
\end{abstract}

Palavras-chave: correlações genéticas, efeito materno, herdabilidade, modelo animal, ovinos deslanados

\begin{abstract}
Records of birth weights at 196 days of age of 927 lamb progenies of 45 sires and 323 dams of Santa Ines sheep, controlled from 1983 to 2000, were used with purpose of evaluating three models that considered or no the maternal genetic effect and covariance between direct and maternal genetic effects, to estimate variance components and genetic parameters by single and two-trait analyses. The (co)variance components and genetic direct and maternal parameters were estimated by restricted maximum likelihood methods, under animal model. The model that included additive direct and maternal effects showed higher value for log likelihood for all the studied traits. Model without maternal effect overestimated the variances and heritability for direct genetic effect (0.56 to 0.23). The importance of maternal effect decreased with growth, as the age of the lambs increased. The variances and heritability
\end{abstract}

Recebido em 31 de outubro de 2004

Aceito em 21 de dezembro de 2005

E-mail: lindenbergsarmento@yahoo.com.br

Apoio: EMEPA-PB e CNPq 
estimates in two-trait models for genetic direct genetic effect were higher than those obtained by singletrait models. The genetic correlations between the traits were high and positive. The maternal effect was important for all the studied traits. The two-trait models allowed better estimate of the direct additive variance, causing higher heritability value.

Keywords: animal model, genetic correlations, hair sheep, heritability, maternal effect

\section{INTRODUÇÃO}

A avaliação genética e a posterior seleção dos animais dependem de vários fatores, dentre eles, a disponibilidade de estimativas de parâmetros genéticos para as características de interesse. No que se refere a ovinos deslanados, essas estimativas são ainda escassas na literatura nacional. Estimativas de parâmetros genéticos separando os efeitos genéticos aditivos direto e materno para características de crescimento são ainda em menor número, sendo basicamente as obtidas nos estudos realizados por Sousa et al. (1999).

As principais informações para avaliação genética e seleção de ovinos de corte são os pesos corporais mensurados durante o período de crescimento. As pesagens são, normalmente, feitas a intervalos de 28 dias, permitindo que cada animal tenha várias medições de seu peso. Os pesos em determinadas idades apresentam características diferentes, geralmente correlacionadas, o que torna fundamental conhecer a magnitude e a direção de tais correlações, uma vez que a seleção de uma delas poderá promover mudanças nas demais.

A estimação de componentes de variância e parâmetros genéticos para essas características pode ser realizada por meio de vários modelos. $\mathrm{O}$ mais simples seria um modelo de repetibilidade, em que todos os pesos medidos ao longo do tempo representassem a mesma característica. Entretanto, esse modelo pressupõe homogeneidade de (co)variâncias genéticas e não-genéticas. Sakaguti (2000), ao estudar o crescimento de bovinos da raça Tabapuã, verificou que o modelo de repetibilidade mostrou-se inadequado à estimação de parâmetros genéticos. Outra forma seria por meio de modelos uni ou multicaracterísticas, assumindo-se que cada peso é uma característica diferente. No modelo multicaracterísticas, as covariâncias entre os pesos nas diferentes idades variam, e as correlações podem ser diferentes da unidade, ou seja, as correlações são levadas em consideração na análise.

O estudo detalhado do crescimento, usando os pesos em várias idades em análise conjunta, por meio de modelos multicaracterísticas, é, em geral, mais consistente quando comparado a um modelo unicaracterística, pois considera as relações entre as características. Em análises multicaracterísticas, a avaliação de um animal para uma característica se faz pela contribuição de todas as outras características envolvidas na análise (Schaeffer, 1984).

A decisão por modelos multicaracterísticas, ao invés de unicaracterística, irá depender, principalmente, das herdabilidades e correlações entre as características, pois conforme a combinação entre tais parâmetros, a eficiência entre esses dois modelos pode ser semelhante. Entretanto, independente do aumento de eficiência, poderá haver outras razões para escolha do modelo multicaracterísticas, como a remoção do viés causado pela seleção seqüencial. Marques et al. (2001), ao analisarem pesos de bovinos, verificaram que a seleção para pesos na desmama e com um ano de idade, usando informações combinadas de ambas as características, seria mais eficiente que com parâmetros estimados por meio do modelo unicaracterística. Segundo esses autores, a escolha de modelos multicaracterísticas no melhoramento animal tem o objetivo de alcançar maior eficiência na resposta à seleção, por utilizar de modo mais completo as informações disponíveis, considerar a existência de valores ausentes, ocasionados pela seleção seqüencial, empregar as correlações existentes entre as características estudadas. Dessa forma, nos últimos anos, os pesquisadores têm dado maior ênfase à análise de dados por meio de modelos multicaracterísticas para estimação dos componentes de variância.

Este trabalho teve como objetivos avaliar três modelos unicaracterística para analisar a 
importância do efeito genético materno e da covariância entre os efeitos genéticos direto e materno, estimar componentes de variância e parâmetros genéticos para pesos de ovinos Santa Inês e comparar os resultados aos obtidos com um modelo bicaracterística.

\section{MATERIAL E MÉTODOS}

Os dados utilizados foram 927 pesos corporais de cordeiros, filhos de 45 reprodutores e 323 matrizes de um rebanho experimental de ovinos da raça Santa Inês, criados em uma fazenda experimental localizada no município de Soledade, semi-árido paraibano, relativos ao período de 1983 a 2000.

Os animais permaneceram em pastagens nativa e nativa melhorada, formadas de capim-buffel
(Cenchrus ciliaris) e capim-pangola (Digitaria decumbens), durante a estação de disponibilidade de alimentos. $\mathrm{Na}$ estação de escassez, os animais receberam, no cocho, suplementação alimentar à base de silagem, palma forrageira (Opuntia ficus sp.), capim-elefante (Peninsetum purpureum) e uma mistura concentrada.

$\mathrm{O}$ arquivo de dados constava de 1261 animais com registros de pesos em várias idades. Com o intuito de tornar os dados mais consistentes, foram feitas algumas restrições, considerando para análise apenas informações de reprodutores com no mínimo três filhos; ovelhas com no mínimo dois filhos; e somente informações de animais nascidos de partos simples e duplo. Definiu-se também que os grupos contemporâneos deveriam incluir, no mínimo, quatro animais. Na Tab. 1 são apresentadas a distribuição do número de observações após as restrições e algumas estatísticas descritivas para as características estudadas.

Tabela 1. Número de observações (n), média e desvio-padrão para as características estudadas

\begin{tabular}{lccc}
\hline Características & $\mathrm{n}$ & \multicolumn{1}{c}{ Média (kg) } & Desvio-padrão (kg) \\
\hline Peso ao nascer (PN) & 855 & 3,61 & 0,72 \\
Peso aos 56 dias de idade (P56) & 924 & 12,65 & 3,32 \\
Peso aos 112 dias de idade - desmame (P112) & 896 & 19,49 & 4,76 \\
Peso aos 168 dias de idade (P168) & 843 & 22,22 & 5,34 \\
Peso aos 196 dias de idade (P196) & 827 & 23,20 & 5,51 \\
\hline
\end{tabular}

Os componentes de (co)variâncias e os parâmetros genéticos foram estimados pelo programa MTDFREML, desenvolvido por Boldman et al. (1995), que utilizaram a metodologia da máxima verossimilhança restrita (REML), por meio de um algoritmo nãoderivativo (Graser et al., 1987), empregando modelos animais em análises uni e bicaracterísticas. O programa utiliza o algoritmo simplex para localizar o mínimo de $-2 \log \mathrm{L}$ ( $\mathrm{L}=$ função de verossimilhança). Os componentes de (co)variância que minimizam $-2 \quad \log \mathrm{L}$ são estimativas de máxima verossimilhança, que maximizam L. Como critério de convergência, utilizou-se a variância dos valores do simplex (-2 $\log \mathrm{L}$ ) inferiores a $10^{-9}$.

Os modelos unicaracterística para estimar os componentes de variância para as características $\mathrm{PN}, \mathrm{P} 56, \mathrm{P} 112, \mathrm{P} 168$ e P196 variaram quanto à inclusão do efeito genético materno, considerando a covariância genética entre os efeitos direto e materno igual ou diferente de zero, como se segue:
Modelo 1: $\mathrm{y}=\mathrm{X} \beta+\mathrm{Z} \mathrm{a}+\varepsilon ;$

$V\left[\begin{array}{l}a \\ \varepsilon\end{array}\right]=\left[\begin{array}{cc}A \sigma_{a}^{2} & 0 \\ 0 & I \sigma_{e}^{2}\end{array}\right]$

Modelo 2: $y=X \beta+Z_{1} a+Z_{2} m+\varepsilon$;

$$
V\left[\begin{array}{l}
a \\
m \\
\varepsilon
\end{array}\right]=\left[\begin{array}{ccc}
A \sigma_{a}^{2} & 0 & 0 \\
0 & A \sigma_{m}^{2} & 0 \\
0 & 0 & I \sigma_{e}^{2}
\end{array}\right]
$$

Modelo 3: $y=X \beta+Z_{1} a+Z_{2} m+\varepsilon$;

$$
V\left[\begin{array}{c}
a \\
m \\
\varepsilon
\end{array}\right]=\left[\begin{array}{ccc}
A \sigma_{a}^{2} & \sigma_{a m} & 0 \\
\sigma_{a m} & A \sigma_{m}^{2} & 0 \\
0 & 0 & I \sigma_{e}^{2}
\end{array}\right]
$$

em que $y=$ vetor $\mathrm{n} \times 1$ de observações; $\mathrm{X}=$ matriz $\mathrm{n} x \mathrm{f}$ de incidência, relacionando as observações aos efeitos fixos; $\beta=$ vetor $\mathrm{f} \times 1$ de efeitos fixos; $Z_{1}=$ matriz $\mathrm{n} \times \mathrm{N}$ de incidência, relacionando as observações aos efeitos genéticos aditivos diretos; $a=$ vetor $\mathrm{N}$ x 1 de efeitos genéticos aditivos diretos; $Z_{2}=$ matriz $\mathrm{n}$ 
$\mathrm{x} \mathrm{N}$ de incidência, relacionando as observações aos efeitos genéticos aditivos maternos; $m=$ vetor $\mathrm{N} \times 1$ de efeitos genéticos aditivos maternos; $\varepsilon=$ vetor de resíduos de mesma dimensão de $y ; \mathrm{A}=$ matriz de numeradores do coeficiente de parentesco entre os indivíduos, de ordem igual ao número de indivíduos (1.257); $\sigma_{a}^{2}=$ variância genética aditiva direta; $\sigma_{m}^{2}=$ variância genética aditiva materna; $\sigma_{a m}=$ covariância entre os efeitos genéticos aditivos direto e materno; $\mathrm{I}_{\mathrm{n}}=$ matriz identidade de ordem $\mathrm{n}$; e $\sigma_{\varepsilon}^{2}=$ variância residual; $\mathrm{N}=$ número de indivíduos; $\mathrm{n}=$ número total de observações; e $\mathrm{f}$ = número de classes de efeitos fixos.

$\mathrm{O}$ efeito de ambiente permanente materno não foi considerado neste estudo. Em análises preliminares, verificou-se que, ao incluir esse efeito, a convergência no processo iterativo foi dificultada, e valores confusos foram estimados, possivelmente em razão do volume de dados disponível.

Os efeitos fixos considerados na análise foram o grupo de contemporâneos, formados pela combinação do ano com a estação de nascimento, o tipo de nascimento, classes de idade da ovelha ao parto (que constituíram sete classes, sendo a primeira classe constituída por fêmeas com idade menor ou igual a dois anos e as demais classes acrescida de um ano, exceto a última que foi constituída por aquelas com mais de sete anos) e o sexo da cria.

Para determinar a importância da inclusão do efeito materno e da covariância entre os efeitos genéticos aditivos direto e materno igual ou diferente de zero no modelo de estimação dos componentes de variância, utilizou-se o teste de razão de verossimilhança (LR). O efeito foi considerado expressivo quando a sua inclusão causava aumento significativo no $\log$ de L. A estatística LR foi obtida pela seguinte expressão: $\mathrm{LR}_{\mathrm{ij}}=2 \log _{\mathrm{e}} \mathrm{L}_{\mathrm{i}}-2 \log _{\mathrm{e}} \mathrm{L}_{\mathrm{j}}$, em que $\mathrm{L}_{\mathrm{i}}=\log \mathrm{L}$ para o modelo completo $\mathrm{i}$; e $\mathrm{L}_{\mathrm{j}}=\log \mathrm{L}$ para o modelo reduzido j. A estimativa LR foi comparada com o valor do qui-quadrado $\left(\chi_{\text {tab }}^{2}\right)$, com um grau de liberdade e nível de significância de $1 \%$. As conclusões foram feitas da seguinte maneira: se LR $>\chi_{(0,01 ; 1)}^{2}$ o teste era significativo, e o modelo completo forneceria maior valor do $\log \mathrm{L}$ em relação ao modelo reduzido. A hipótese de nulidade testada foi de que as funções dos modelos completo e reduzido não diferiam entre si.

As análises bicaracterísticas foram executadas entre os pares de pesos considerados no estudo, usando o modelo indicado LR, com base nos resultados das análises com os modelos unicaracterística, levando-se em consideração uma distribuição conjunta entre os pares de características. As estimativas dos componentes de variância para determinada característica foram calculadas como a média de todas as análises bicaracterísticas que incluíram a referida característica.

\section{RESULTADOS E DISCUSSÃO}

As estimativas dos componentes de variância para as características estudadas obtidas pelos modelos unicaracterística, bem como os valores do logaritmo da função de verossimilhança (log L) para cada modelo, estão sumarizadas na Tab. 2.

A inclusão do efeito genético materno (modelo 2) proporcionou aumento nos valores do $\log \mathrm{L}$ para as características, quando comparados com os valores obtidos pelo modelo 1 (Tab.2), sendo estes aumentos estatisticamente significativos pelo teste LR. No modelo 3, quando se considerou a covariância entre os efeitos genéticos aditivos direto e materno diferente de zero, pequena mudança no $\log \mathrm{L}$ foi verificada, não sendo significativa para nenhuma das características, podendo ser indicativo de que os efeitos genéticos direto e materno podem ser assumidos como não correlacionados, corroborando, assim, os achados de Sousa et al. (1999).

Os valores do teste LR para o modelo 2 (Tab. 2) decresceram a partir do peso aos 56 dias de idade, evidenciando diminuição da importância desse efeito nos pesos após o desmame. Esses resultados indicam que a inclusão do efeito genético materno nos modelos de análise é fundamental mesmo para pesos pós-desmama, como aos 168 e 196 dias de idade.

As estimativas dos parâmetros genéticos para as características estudadas estão apresentadas na Tab. 3. 
Tabela 2. Estimativas de componentes de variância, valores do logaritmo da função de verossimilhança $\left(\log _{\mathrm{e}} \mathrm{L}\right)$ e valores do teste de razão de verossimilhança (LR) para as características estudadas, de acordo com o modelo de análise

\begin{tabular}{ccccccc}
\hline Modelo & Parâmetro & PN & P56 & P112 & P168 & P196 \\
\hline \multirow{4}{*}{1} & $\hat{\sigma}_{a}^{2}$ & 0,22199 & 2,86189 & 5,38941 & 5,18632 & 3,97282 \\
& $\hat{\sigma}_{e}^{2}$ & 0,17598 & 3,39117 & 7,00848 & 12,94667 & 13,44066 \\
& $\hat{\sigma}_{f}^{2}$ & 0,39796 & 6,25306 & 12,39789 & 18,13300 & 17,41348 \\
& $\log _{\mathrm{e}} \mathrm{L}$ & $-17,8754$ & $-1201,403$ & $-1445,413$ & $-1517,383$ & $-1483,918$ \\
\hline \multirow{4}{*}{2} & $\hat{\sigma}_{a}^{2}$ & 0,06086 & 0,00005 & 0,00007 & 0,05101 & 0,00003 \\
& $\hat{\sigma}_{m}^{2}$ & 0,07906 & 1,65545 & 2,81756 & 3,45788 & 3,04825 \\
& $\hat{\sigma}_{e}^{2}$ & 0,23586 & 4,24992 & 8,88388 & 14,10753 & 13,99528 \\
& $\hat{\sigma}_{f}^{2}$ & 0,37577 & 5,90537 & 11,70144 & 17,61643 & 17,04356 \\
& $\log _{\mathrm{e}} \mathrm{L}$ & $-7,1582$ & $-1181,749$ & $-1432,409$ & $-1508,117$ & $-1475,464$ \\
$\mathrm{LR}^{1}$ & $21,4343^{* *}$ & $39,3084^{* *}$ & $26,0083^{* *}$ & $18,5310^{* *}$ & $16,9093^{* *}$ \\
\hline & $\hat{\sigma}_{a}^{2}$ & 0,07571 & 0,00004 & 0,00006 & 0,30977 & 0,00374 \\
& $\hat{\sigma}_{m}^{2}$ & 0,11917 & 1,657 & 2,81756 & 2,51404 & 3,02024 \\
& $\hat{\sigma}_{a m}$ & $-0,04467$ & 0,00075 & $-0,0006$ & 0,88248 & 0,10625 \\
& $\hat{\sigma}_{e}^{2}$ & 0,22719 & 4,24948 & 8,88388 & 13,92502 & 13,96134 \\
& $\hat{\sigma}_{f}^{2}$ & 0,37741 & 5,90723 & 11,70084 & 17,6313 & 17,09157 \\
& $\log _{\mathrm{e}} \mathrm{L}$ & $-6,8404$ & $-1181,749$ & $-1432,409$ & $-1507,706$ & $-1475,406$ \\
$\mathrm{LR}^{2}$ & $0,6356^{\mathrm{ns}}$ & $0,0008^{\mathrm{ns}}$ & $-0,0003^{\mathrm{ns}}$ & $0,8226^{\mathrm{ns}}$ & $0,1162^{\mathrm{ns}}$ \\
\hline
\end{tabular}

${ }^{* *}=$ significativo a $1 \%$ de probabilidade; $\mathrm{ns}=$ não significativo ${ }^{1}{ }^{1}=$ modelo $2 \operatorname{com~} 1 ;{ }^{2}=$ modelo $3 \operatorname{com} 2$.

$\hat{\sigma}_{a}^{2}=$ variância genética aditiva direta; $=\hat{\sigma}_{e}^{2}$ variância residual; $\hat{\sigma}_{f}^{2}=$ componente de função quadrática dos efeitos fixos; $\hat{\sigma}_{m}^{2}=$ variância genética aditiva materna; $\hat{\sigma}_{a m}=$ covariância dos efeitos genéticos aditivos direto e materno.

Tabela 3. Herdabilidades para efeitos genéticos aditivos direto $\left(\hat{h}_{a}^{2}\right)$ e materno $\left(\hat{h}_{m}^{2}\right)$ e correlações entre os efeitos aditivos direto e materno $\left(r_{a m}\right)$ para as características estudadas, de acordo com o modelo de análise

\begin{tabular}{lcccccc}
\hline Modelo & Parâmetro & PN & P56 & P112 & P168 & P196 \\
\hline 1 & $\hat{h}_{a}^{2}$ & 0,56 & 0,46 & 0,43 & 0,29 & 0,23 \\
\hline 2 & $\hat{h}_{a}^{2}$ & 0,16 & 0,00 & 0,00 & 0,00 & 0,00 \\
& $\hat{h}_{m}^{2}$ & 0,21 & 0,28 & 0,24 & 0,20 & 0,18 \\
\hline \multirow{3}{*}{3} & $\hat{h}_{a}^{2}$ & 0,20 & 0,00 & 0,00 & 0,02 & 0,00 \\
& $\hat{h}_{m}^{2}$ & 0,32 & 0,28 & 0,24 & 0,14 & 0,18 \\
& $r_{a m}$ & $-0,47$ & 0,99 & $-0,24$ & 1,00 & 1,00 \\
\hline
\end{tabular}

As estimativas das variâncias genéticas aditivas diretas e das herdabilidades para as características estudadas obtidas pelo modelo 1 foram consistentemente mais altas do que as estimadas pelos modelos 2 e 3 (Tab. 2 e 3 ). Duguma et al. (2002) verificaram resultados semelhantes, nos quais as herdabilidades estimadas por meio de modelos que não incluíram o efeito materno foram maiores. Essas estimativas foram superiores à maioria daquelas relatadas por vários autores para diferentes raças de ovinos deslanados e métodos de estimação 
(Silva et al., 1996; Sousa et al., 1999; Quesada et al., 2002).

Nos modelos 2 e 3 , nos quais se incluiu, além do efeito aditivo direto, o efeito materno (modelo 2) e o efeito materno e a covariância entre os efeitos direto e materno diferente de zero (modelo 3 ), as estimativas de herdabilidade para os efeitos genéticos aditivos diretos foram mais baixas para as cinco características estudadas, quando comparadas às obtidas pelo modelo 1 . Essa mesma tendência foi constatada por Sousa et al. (1999), ao ajustarem um modelo correspondente ao 3 para as características PN, P112 e P196 em ovinos da raça Santa Inês. Resultados similares foram reportados por Duguma et al. (2002), os quais, ao incluírem o efeito materno no modelo, observaram que as estimativas de herdabilidade direta diminuíram de 0,38 para 0,19 no $\mathrm{PN}$ e de 0,36 para 0,29 no peso ao desmame. Esses resultados evidenciam que a não-inclusão do efeito genético materno no modelo de análise para características de crescimento de ovinos pode inflacionar os componentes de variância devido aos efeitos genéticos aditivos diretos, resultando em estimativas de herdabilidades superestimadas.

As herdabilidades aditivas diretas estimadas pelos modelos 2 e 3, com exceção do $\mathrm{PN}$, foram próximas de zero. Esses valores podem ter sido obtidos devido ao tamanho da amostra, ou seja, o pequeno número de observações consideradas neste estudo, possivelmente, dificultou a partição das variâncias entre os efeitos direto e materno pelo método empregado. Dessa forma, ao incluir o efeito genético materno no modelo, a variação decorrente do efeito genético aditivo direto pode ter sido direcionada para o efeito genético materno, fazendo com que os componentes de variâncias e herdabilidades estimadas para os efeitos aditivos diretos ficassem próximos de zero. Sousa et al. (1999), ao incluir o efeito genético materno no modelo, constatou que a herdabilidade para o efeito genético direto diminuiu de 0,37 para 0,04 e de 0,31 para 0,06 para P112 e P196, respectivamente.

As estimativas de herdabilidade para o efeito genético materno foram de magnitude média, variando de 0,28 para P56 a 0,18 para P196. Mesmo o efeito genético materno permanecendo evidente após a desmama, verificou-se tendência de declínio, concordando com a maioria dos resultados encontrados na literatura (Sousa et al., 1999; El Fadili et al., 2000; Quesada et al., 2002). Essa tendência era, de certa forma, esperada, pelo fato de o cordeiro tornar-se menos dependente da mãe à medida que cresce.

Com a desmama acontecendo por volta dos 112 dias de idade, esperava-se que aos 196 dias de idade a influência materna fosse de menor magnitude. O resultado observado pode ser devido às condições de criação, em que os cordeiros são criados ao pé da ovelha, favorecendo uma maior dependência destes aos cuidados maternos ou pelo fato de as variâncias terem sido particionadas erroneamente entre os efeitos genéticos. Dessa forma, mesmo os cordeiros sendo totalmente desmamados aos 112 dias de idade, uma influência residual desse efeito pode ter se estendido até P196. Estudos adicionais com pesos medidos em idades mais avançadas e com um maior volume de dados devem ser conduzidos, visando buscar a informação sobre até que idade a influência desse efeito pode persistir. Além das causas citadas anteriormente, vale salientar também que, pelo fato de não ter incluído nos modelos o efeito de ambiente permanente materno, a variância genética aditiva materna pode ter sido inflacionada, resultando em variâncias e herdabilidades maternas superestimadas.

As estimativas de correlações entre os efeitos genéticos direto e materno foram moderadas e negativas para PN e P112, porém altas e positivas para os demais pesos (Tab. 3). Sousa et al. (1999) encontraram correlações de $-0,15$ e 0,31 para PN e P112, respectivamente, e de 0,67 para P196. Os resultados encontrados na literatura para pesos pós-desmama mostraram uma tendência de essa correlação ser positiva de média a alta magnitude, mas não igual à unidade, como encontrado neste estudo, o que pode ser reflexo do número de informações analisadas.

Muitas discussões têm sido feitas sobre o sinal da correlação entre os efeitos direto e materno em estudos com bovinos de corte. Para Robinson (1996), as estimativas negativas são provavelmente conseqüência de uma variação adicional entre touros, ou variação reprodutor $\mathrm{x}$ ano, do que evidências de um relacionamento genético negativo verdadeiro. Eler et al. (2000) verificaram que as correlações passaram de negativas para próximas de zero e positivas 
quando se considerou, no modelo, o efeito da interação reprodutor $\mathrm{x}$ rebanho. No caso de ovinos de corte, os resultados observados são de certo modo contraditórios. Maria et al. (1993) encontraram correlações altas e negativas entre esses efeitos para vários pesos, Neser et al. (2001) estimaram correlação positiva e moderada para PN e de moderada a alta, mas negativa para peso à desmama, Duguma et al. (2002) obtiveram estimativas negativas e moderadas para $\mathrm{PN}$ e baixas e positivas para peso à desmama e Boujenane e Kansari (2002) verificaram correlações moderadas e negativas para características do nascimento aos 90 dias de idade.

As análises com os modelos bicaracterísticas, combinando as cinco características consideradas, foram realizadas utilizando-se $\mathrm{o}$ modelo 2, uma vez que o teste LR indicou que o efeito genético materno deve ser considerado no modelo para estimação de componentes de variâncias e parâmetros genéticos para as características de crescimento de ovinos Santa Inês. Os resultados obtidos nessas análises estão apresentados na Tab. 4

Tabela 4. Estimativas de herdabilidade média para os efeitos genéticos aditivos direto (diagonal fora de parênteses), materno (diagonal entre parênteses) e de correlações genéticas (acima da diagonal) e fenotípicas (abaixo da diagonal) para as características estudadas

\begin{tabular}{lccccc}
\hline Características & PN & P56 & P112 & P168 & P196 \\
PN & $0,23(0,17)$ & 1,00 & 1,00 & 0,75 & 1,00 \\
P56 & 0,24 & $0,06(0,25)$ & 1,00 & 1,00 & 1,00 \\
P112 & 0,21 & 0,42 & $0,03(0,23)$ & 0,99 & 1,00 \\
P168 & 0,19 & 0,41 & 0,46 & $0,05(0,19)$ & 1,00 \\
P196 & 0,20 & 0,38 & 0,44 & 0,55 & $0,03(0,18)$ \\
\hline
\end{tabular}

$\mathrm{PN}=$ peso ao nascer; $\mathrm{P} 56=$ peso aos 56 dias..., $\mathrm{P} 196=$ peso aos 196 dias de idade.

As estimativas de herdabilidade direta foram levemente superiores, para as cinco características, às obtidas pelo modelo 2 nas análises com os modelos unicaracterística. Podese observar na Fig. 1 que a herdabilidade para o efeito direto tendeu a diminuir do nascimento aos 196 dias de idade, sendo a maior redução verificada do nascimento aos 56 dias: de 0,23 para 0,06. Esses resultados sugerem que a informação de outra característica contribuiu na estimação das variâncias entre os efeitos genéticos aditivos diretos e, de certa forma, até melhorou a partição dos efeitos genéticos direto e materno. Aumento de herdabilidade com modelos bicaracterísticas foi relatado por Torres (1998). Além das vantagens citadas por Marques et al. (2001), Torres (1998) enfatiza que tal aumento tenha ocorrido, provavelmente, devido ao incremento no número de observações em análises bicaracterísticas, que utilizam a relação entre animais nas diferentes características para estimar esses valores, contribuir para resgatar maior proporção da variância genética aditiva.

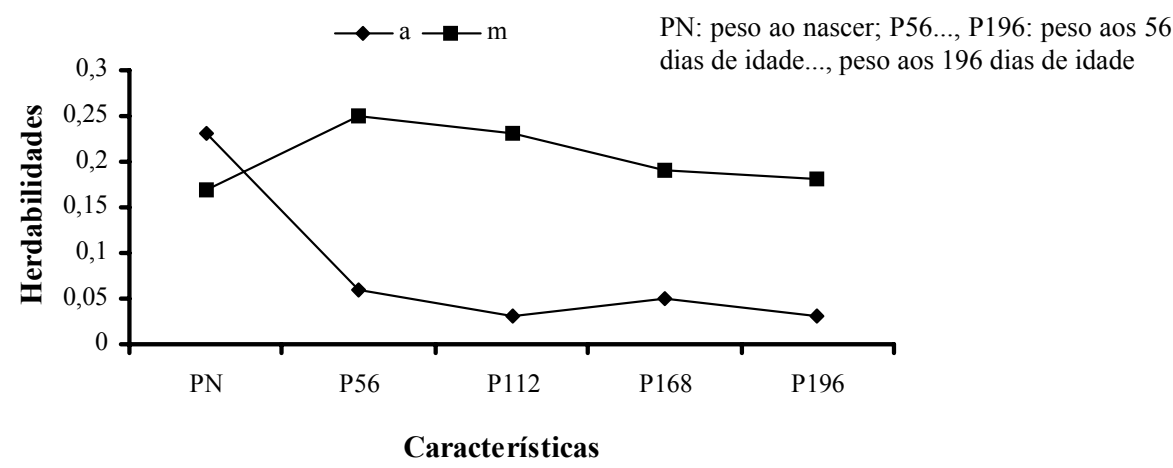

Figura 1 - Estimativas de herdabilidades direta (a) e materna (m) para as características avaliadas durante o período de crescimento estudado. 
Sousa et al. (1999) estimaram herdabilidade de $0,09,0,05$ e 0,08 , por meio de modelos bicaracterísticas, para PN, P112 e P196, respectivamente, havendo pequena redução na estimativa de herdabilidade para PN e ligeiro aumento nas estimativas dos demais pesos, quando comparadas às obtidas pelo modelo unicaracterística. Boujenane e Kansari (2002) obtiveram com os modelos bicaracterísticas estimativas de $0,05,0,02,0,06$ e 0,06 para os pesos ao nascer, 30, 70 e 90 dias de idade, não havendo, praticamente, alteração quando comparadas com as herdabilidades estimadas por meio de modelos unicaracterística.

De modo geral, as estimativas de herdabilidade direta para as características foram de baixa magnitude. Esses resultados mostram que o fenótipo não é um bom indicador do genótipo dos indivíduos. Assim, espera-se que a seleção individual não proporcione ganhos genéticos satisfatórios. Vale salientar que esses resultados têm que ser vistos com certa cautela, pois os mesmos foram estimados a partir de um conjunto pequeno de dados, sendo importante a realização de estudos com maior volume de dados, de modo a confirmar ou não resultados entrados no presente estudo.

As estimativas de herdabilidade materna apresentaram o mesmo comportamento das obtidas pelos modelos unicaracterística, porém com valores ligeiramente inferiores (Tab. 4). Observa-se na Fig. 1 que houve pequeno aumento até os 56 dias de idade e, a partir desse ponto, decréscimo até os 196 dias. Assim como nos modelos unicaracterística, a contribuição da parte genética materna ainda permaneceu alta aos 196 dias, com uma herdabilidade de 0,18, confirmando que nessa idade existem resquícios desse efeito ou, ainda, que o aumento no número de informações, com os modelos bicaracterísticas, não foi totalmente eficiente na correta partição dos efeitos genéticos direto e materno. Boujenane e Kansari (2002) estimaram herdabilidades para o efeito materno iguais a $0,05,0,07,0,07$ e 0,04 para os pesos ao nascer, 30,70 e 90 dias, respectivamente, sendo essas estimativas semelhantes às obtidas com os modelos unicaracterística.

As estimativas de correlações genéticas diretas entre as características foram todas positivas e de alta magnitude - quase todas iguais à unidade
(Tab. 4). Esses resultados não mostraram uma tendência esperada, isto é, de diminuir à medida que a distância em tempo entre as pesagens aumenta. Isso pode ter acontecido, provavelmente, devido ao pequeno número de informações disponíveis para estimar essas correlações. Os valores encontrados na literatura são na maioria inferiores aos obtidos neste estudo, como os verificados por Sousa et al. (1999), que estimaram correlações de 0,37 entre PN e P112; de -0,11 entre PN e P196; e de 0,28 entre P112 e P196. Quesada et al. (2002) obtiveram correlações positivas variando de 0,21 a 0,39 entre os pesos do nascimento aos 210 dias de idade em ovinos Morada Nova, as quais tenderam a diminuir, ao passo que as pesagens se distanciaram no tempo. Essa mesma tendência foi verificada por Boujenane e Kansari (2002), porém com valores de maior magnitude, variando de 0,69 a 1,00 para os pesos do nascimento aos 90 dias de idade.

As altas correlações genéticas obtidas neste estudo entre as características indicam que a maioria dos genes que atua em uma atua nas demais e no mesmo sentido; disso resulta que os animais mais pesados ao desmame também o podem ser aos 196 dias de idade, por exemplo. No entanto, sabe-se que a resposta correlacionada não depende exclusivamente da correlação genética entre as características, mas também das herdabilidades das características envolvidas no processo de seleção. Dessa forma, apesar das altas correlações genéticas entre as características, a resposta correlacionada pela seleção indireta, mesmo sendo eficiente, resultará em ganhos genéticos muito baixos.

As correlações fenotípicas obtidas foram todas positivas e variaram de baixa a moderada magnitude, sendo as mais altas entre idades adjacentes (Tab. 4), indicando que as características estudadas têm certa dependência fenotípica. Os resultados obtidos por Boujenane e Kansari (2002) seguiram essa mesma tendência, porém com valores de maior magnitude, variando de 0,28 a 0,91 entre pesos do nascimento aos 90 dias de idade.

\section{CONCLUSÕES}

Os valores de herdabilidades obtidas para os pesos corporais nas idades consideradas indicam 
que o progresso genético esperado por meio de seleção individual é pequeno. $\mathrm{O}$ efeito genético materno no modelo apresentou importante influência sobre todas as características estudadas, devendo, portanto, ser considerado nos estudos de características de crescimento, mesmo após a desmama. As análises bicaracterísticas, por utilizarem maior número de informações e as relações entre as características, possibilitaram resgatar parte da variância aditiva direta, levando a estimativas maiores de herdabilidade. Estudos adicionais devem ser realizados envolvendo maior número de informações, o que permitirá inferir com maior exatidão esses parâmetros.

\section{REFERÊNCIAS BIBLIOGRÁFICAS}

BOLDMAN, K.G.; KRIESE, L.A.; Van VLECK, D.L. et al. A manual for use of MTDFREML. A set of programs to obtain estimates of variances and covariances [DRAFT] Lincoln: USDA/ARS, 1995. 120p.

BOUJENANE, I.; KANSARI, J. Estimates of (co)variances due to direct and maternal effects for body weights in Timahdite sheep. Anim. Sci., v.28, p.409-414, 2002.

DUGUMA, G.; SCHOEMAN, S.J.; CLOETE, S.W.P. et al. Genetic parameter estimates of early growth traits in the Tygerhoek Merino flock. S. Afr. J. Anim. Sci., v.32, p.66-75, 2002.

EL FADILI, M.; MICHAUX, C.; DETILLEUX, P.L. et al.Genetic parameters for growth traits of the Moroccan Timahdit breed of sheep. Small Rumin. Res., v.37, p.203-208, 2000.

ELER, J.P.; FERRAZ, J.B.S.; GOLDEN, B.L. et al. Influência da interação touro $\mathrm{x}$ rebanho na estimação da correlação entre efeitos genéticos direto e materno em bovinos da raça Nelore. Rev. Bras. Zootec., v.29, p.1642-1648, 2000.

GRASER, H.U.; SMITH, S.P.; TIER, B. A derivative-free approach for estimating variance components in animal model by restricted maximum likelihood. J. Anim. Sci., v.64, p.13621370, 1987.

MARIA, G.A.; BOLDMAN, K.G.; VAN VLECK, L.D. Estimates of variance due to direct and maternal effects for growth traits of
Romanov sheep. J. Anim. Sci., v.71, p.845-849, 1993.

MARQUES, L.F.A.; OLIVEIRA, H.N.; PEREIRA, J.C.C. Análises uni e bicaracterística dos pesos de bovinos Simental. In: REUNIÃO ANUAL DA SOCIEDADE BRASILEIRA DE ZOOTECNIA, 38., 2001, Piracicaba. Anais... Piracicaba: SBZ, 2001. CD-ROM. Melhoramento Animal.

NESER, F.W.C.; ERASMUS, G.J.; VAN WYK, J.B. Genetic parameter estimates for pre-weaning weight traits in Dorper sheep. Small Rumin. Res., v.40, p.197-202, 2001.

QUESADA, M.; MCMANUS, C.; COUTO, F.A.A. Efeitos genéticos e fenotípicos sobre características de produção e reprodução de ovinos deslanados no Distrito Federal. Rev. Bras. Zootec., v.31, p.342-349, 2002.

ROBINSON, D.L. Estimation and interpretation of direct and maternal genetic parameters for weights of Australian Angus cattle. Livest. Prod. Sci., v.45, p.1-11, 1996.

SAKAGUTI. Funções de covariâncias e modelos de regressão aleatória na avaliação genética do crescimento de bovinos jovens da raça Tabapuã. 2000. 81f. Tese (Doutorado) Universidade Federal de Viçosa, Viçosa, MG.

SCHAEFFER, L.R. Sire and cow evaluation under multiple trait models. J. Dairy Sci., v.67, p.1567-1580, 1984.

SILVA, F.L.R.; FIGUEIREDO, E.A.P.; SIMPLÍCIO, A.A. et al. Parâmetros genéticos e fenotípicos de características de crescimento de desmame de cordeiros Santa Inês, no Estado do Ceará. Rev. Bras. Zootec., v.25, p.845-852, 1996.

SOUSA, W.H.; PEREIRA, C. S.; BERGMANN, J.A.G. et al. Estimativas de componentes de (co)variância e herdabilidade direta e materna de pesos corporais em ovinos da raça Santa Inês. Rev. Bras. Zootec., v.28, p.1252-1262, 1999.

TORRES, R.A. Efeito da heterogeneidade de variância na avaliação genética de bovinos da raça Holandesa no Brasil. 1998. 124f. Tese (Doutorado) - Escola de Veterinária, Universidade Federal de Minas Gerais, Belo Horizonte, MG. 